\title{
Anti-Neutrophil Cytoplasmic Antibodies In Systemic Lupus Erythematosus And Lupus Nephritis: An Overlapping Syndrome
}

\author{
Tahmina Jesmin ${ }^{1}$,Ranjit Ranjan Roy ${ }^{1}, M d$. Firoz Anjum ${ }^{2}$, Muslima Akter ${ }^{2}$ \\ Department Of Pediatric Nephrology, Bangabandhu Sheikh Mujib Medical University, Dhaka, Bangladesh
}

\begin{abstract}
Systemic lupus erythematosus (SLE) and small-sized vessel vasculitis are usually two distinguishable autoimmune diseases. However, vasculitis may be found in the course of SLE but rarely corresponds to an antineutrophil cytoplasmatic antibodies (ANCA) associated vasculitis (AAV).

We report a case SLE with lupus nepritis (LN) associated with perinuclear (p-ANCA). The frequency of this association seems not fortuitous. Although the etiopathogenic mechanisms of such association remain to be described, several clinical, histological and immunological features support the hypothesis of the existence of SLE-AAV overlapping syndrome. Moreover clinicians must be aware of such overlapping syndrome, notably because its initial presentation can be very severe.
\end{abstract}

Keywords: Systemic lupus erythematosus, antineutrophil cytoplasmatic antibodies, ANCA associated vasculitis.

\section{Introduction}

SLE is a multisystem autoimmune connective tissue disorder of unknown cause that can affect multiple organs ${ }^{1}$. SLE presentation is heterogeneous and can display a broad spectrum of manifestations, including LN in $30 \%-60 \%$ and vasculitis in $11 \%{ }^{2}$. SLE characterized by the presence of multiple autoantibodies: primary (including antinuclear antibody (ANA), circulating immune complexes, and activation of the complement system) or secondary depending on whether they are recognized as having a primary role or otherwise, in the pathogenesis of the disease. ANCA is considered a secondary autoantibody. The prevalence of ANCA is reported to be as high as $31 \%$ in lupus patients ${ }^{3}$. AAV produce distinct pattern of immunofluorescence (IF) staining around the nucleus is known as perinuclear (pANCA), it is associated with antibodies against the enzyme myeloperoxidase (MPO). Diffuse granular staining of the neutrophil cytoplasm is termed as cytoplasmic (cANCA), it corresponds to the enzyme proteinase 3 (PR3) ${ }^{4}$.ANCAs are strongly associated with small vessel vasculitis, which includes Wegener's granulomatosis, microscopic polyangitis, and Churg-Strauss syndrome ${ }^{5}$.

These two diseases are only rarely associated: a vasculitis may occur in the course of SLE but rarely responds to AAV classification criteria. Indeed, when ANCAs are tested positive in the course of SLE, no vasculitis is usually found ${ }^{6}$. It is estimated that in $4 \%$ of SLE patients clinical signs of vasculitis may be found ${ }^{7}$. To the best of our knowledge, the coexistence of SLE and AAV occurs very rarely in children. Here we report a case of SLE with LN who is both ANCA and antiphospholipid antibody positive without any features of vusculitis or thromboembolic manifestations. Purpose of this report is to highlight the importance of doing ANCA in case of SLE

Case: A 13 years old girl, immunized, admitted with fever for one month; generalized swelling and scanty icturation for 10 days. She gave history of multiple joints pain (both wrist and ankle), skin rash, photosensitivity and painless ulcer over hard palate. No history of hematuria, headache, convulsion, respiratory distress, hemoptysis, sinusitis, and such type of illness in family.

On examination: Fretful, edematous, febrile, severely pale, hypertensive, pulse rate and respiratory rate (RR) normal, both weight and height lies on $3^{\text {rd }}$ centile, bed side urine for albumin $2+$. Ooral cavity healthy and healed ulcer over hard palate; abdomen was distended, non tender, hepatomegaly and ascites present, bowel sound present. Both ankle and wrist joint not swollen, tender in both ankle and wrist joint (grade 2), not restricted movement, other joints normal. Other system revealed normal finding.

Immediate investigations showed hemoglobin $5.6 \mathrm{gm} / \mathrm{dl}$, erythrocyte sedimentation rate $68 \mathrm{~mm}$ in $1^{\text {st }}$ $\mathrm{hr}$, total count $9500 / \mathrm{cmm}$, neutrophil $67 \%$, lymphocyte $30 \%$, platelet $20,000 / \mathrm{cmm}$, red blood cell (RBC) $3000 / \mathrm{cmm}$ and peripheral blood film showed microcytic hypochromic anemia. Urine routine microscopic examination (RME): albumin 3+, RBC 25-40/HPF and pus cell 4-8/HPF, granular cast present. Urine culture and sensitivity showed no growth. 24 hours urinary total protein was $3.4 \mathrm{gm} /$ day. Serum albumin $17 \mathrm{gm} / \mathrm{l}$, serum creatinine $0.68 \mathrm{mg} / \mathrm{dl}$, ANA and anti dsDNA positive, C3 and C4 decreased, Direct coombs test negative (as PRBC was given during hospitalization before taking blood sample), pANCA positive, antiphospholipid IgG positive, Prothrombin and activated partial thromboplastin time normal. Ultrasonogram of kidney, ureter and bladder shows acute inflammatory change of kidney with normal size and shape. Renal biopsy revealed diffuse 
proliferative lupus nephritis (class 4).During hospitalization she was treated with fluid and salt restriction, tab hydroxychloroquine,PRBC infusion, antihypertensive along with hematinics. After doing biopsy on $2^{\text {nd }}$ day of admission she was given IV methylprednisolone $30 \mathrm{mg} / \mathrm{kg}$ daily for 3 days followed by oral prednisolone along with IV cyclophosphamide $750 \mathrm{mg} / \mathrm{m}^{2}$ first dose, and she became stable.On $10^{\text {th }}$ days of admission, she developed dyspnea and chest pain. She was restless, RR 32 breaths per minute, other vital were within normal range, cardiovascular examination revealed new onset ejection systolic murmur. Echocardiogram shows moderate pericardial effusion with mild pulmonary hypertension and managed with oxygen inhalation, propped up position and diuretics and became stable.

Patient achieved complete remission after 20 days of starting treatment. During discharge, she adviced to receive oral prednisolone $2 \mathrm{mg} / \mathrm{kg}$ /day (for total 4 weeks followed by tapering) along with pulse IV cyclophosphamide for total 6 months; followed by maintenance therapy and this regimen started after consulting with institutional expert. She was also prescribed low dose aspirin as a prophylaxis to prevent thrombembolism as antiphospholipid antibody was positive. Regular follow up advised given to look for clinical, laboratories status.

\section{Discussion}

SLE and AAV are both rare diseases. The number of cases of SLE associated with AAV reported in the literature is low. Altogether, patients with SLE/AAV overlap syndrome are mostly female, (as our patient was also female). The presentation of AAV is MPA-like in most patients, with articular, cutaneous and renal involvement ${ }^{8}$.

The presence of ANCA in patients with SLE is recognized with reported prevalence rate ranging from $25 \%$ to $56 \% \%^{9}$. Chin et al reported that cANCA $6 \%$ and pANCA $32 \%$ positive in patient with SLE with (LN) ${ }^{10}$. Here, our case also had pANCA positive in SLE with LN.

Kuster et al found ANCA asoociated with lupus nephritis ${ }^{11}$. Our case also diagnosed SLE with lupus nephritis (on the basis of history, physical examination and laboratories) along with pANCA positive. of serositis.

Lee et al found moderate or marginal elevations of anti-MPO in serositis ${ }^{12}$. Our case also had features

Jarrot et al. described the presence of overlapping antibodies in SLE with LN or pauci-immune GN was not associated with the proportion of biopsy finding, which highlights the difference between true SLE/AAV overlap syndrome and the more common finding of ANCA positivity in $\mathrm{LN}^{13}$. Here in our patient's renal histology showed that diffuse proliferative lupus nephritis (class-4) ISN/RPS classification.

Interestingly, SLE may be complicated with antiphospholipid syndrome so antiphospholipid antibody should be examined in patient with recurrent thrombosis, livedo reticularis, chorea, cerebrovascualr accidents and hypertension ${ }^{14}$. However, there was no evidence of such finding in our case, although antiphospholipid IgG was positive, so here just prescribed aspirin as a prophylaxis but should be kept in mind about these complication and appropriate intervention should be given.

However, here we managed the patient as class-4 $\mathrm{LN}^{14}$ as mentioned earlier.

\section{Conclusion}

The association of SLE and AAV is rare. Moreover, in our case reported herein and the literature review argues for the existence of an overlapping syndrome. The clinician must be aware of this association and search for both ANA and ANCA in such cases. Future studies are needed to investigate the possible shared immune pathogenesis that may facilitate the co-occurrence of SLE and AAV in these patients and so lead to new therapeutic options.

Competing interest is none declared and also non-funding.

\section{References}

[1]. Jaybhaye AP, Sutay NR, Chate SV, Rathod TN. Juvenile systemic lupus erythematosus: A diagnostic dilemma. J Nat Sci Biol Med 2011;2:229-31.

[2]. Maroz N, Segal MS. Lupus nephritis and end-stage kidney disease. Am J Med Sci. 2013;346:319-323.

[3]. Fauzi AR, Kong NCT, Chua MK, Jeyabalan V, Idris MN, Azizah R. Antibodies in Systemic Lupus Antineutrophil Cytoplasmic Erythematosus: Prevalence, Disease Activity Correlations and Organ System Associations. Med J Malaysia Vol 59 No 3 August 2004 372-377

[4]. Bitzan M. Glomerular Disease. In: Phadke K, Goodyer P, Bitzan M, editors. Manual of Pediatric Nephrology, $1^{\text {st }}$ ed., Springer, London; 2014. 141-229.

[5]. Bosch X, Guilabert A, Font J. Antineutrophil cytoplasmic antibodies. Lancet 2006; 368:404-18.

[6]. Hervier B, Hamidou M, Haroche J, Durant C, Mathian A, Amoura Z. Systemic lupus erythematosus associated with ANCAassociated vasculitis: an overlapping syndrome? Rheumatol Int 2012; 32:3285-3290

[7]. Kenneth TC, Balabanova M. Vasculitis in systemic lupus erythematosis. Clin Dermatol 2004; 22:148-56.

[8]. Villiger PM, Guillevin L. Microscopic polyangiitis: clinical presentation. Autoimmun Rev. 2010; 9:812-819.

[9]. Davenport A, Lock RJ, Wallington TB, Feest TG. Clinical significant of the serial measurement of autoantibodies to neutrophils cytoplasm using a standard indirect immunofluorescence test. Am J Nephrol 1995; 15: 201-7. 
[10]. Chin HJ, Curie A, SL Chun, Chung HK. Clinical implications of antineutrophil cytoplasmic antibody test in lupus nephritis. Am J Nephrol 2000; 20(1); 57-64.

[11]. Kuster S, Apenberg S, Andrassy K, Ritz E. Antineutrophil cytoplasmic antibodies in SLE. Contrib Nephrol 1992: 99; 94-98.

[12]. Lee SS, Lawton JWM, Chak W. Distinction between antinuclear antibody and pANCA.

a. J Clin Pathol 1991;44 962-62.

[13]. Jarrot PA, Chiche L, Hervier B, Daniel L, Vuiblet V, Bardin N, et al. Systemic Lupus Erythematosus and Antineutrophil Cytoplasmic Antibody-Associated Vasculitis Overlap Syndrome in Patients With Biopsy- Proven Glomerulonephritis. Medicine; 2016; 1-11.

[14]. Gulati A and Bagga A. Sytemic Vasculitis and Lupus Nephritis' In: Srivastava RN, Bagga A, editors. Pediatric Nephrology, $6^{\text {th }}$ ed., Jaypee Brothers Medical Publishers (P) Ltd, New Delhi; 2016. 149-165. 